\title{
Behavior fixations maintained by pain and by fear
}

\section{MORTON H. KLEBAN, PHILADELPHIA GERIATRIC CENTER, 5301 Old York Road, Philadelphia, Pa. 19141}

The paper describes two different types of sterotyped behavior, that produced by intermittent shock (ISh) and that produced by continuous shock (CSh). ISh does not disrupt ongoing behavior as much as CSh, nor does it coerce stereotypy as rapidly as CSh, but recoverability from ISh is much slower than from CSh. This is discussed in terms of fear conditioning and pain stereotypy.

Both pain and fear are capable of acting as strong motivators of behavior. The Sidman procedure is a clear example of how a fear state can sustain behavior for protracted periods. But in many experimental settings, e.g., escape, intermittent escape, etc., pain and fear are combined experiences. Miller describes this as "pain-fear" (1948). Together they act to motivate behavior.

This leads to an interesting question, do the pain and fear components of pain-fear work together to motivate the same behavior? Or, do they motivate different behavioral tendencies? In this paper evidence will be presented to suggest that pain and fear components motivate different behavioral tendencies. The observations supporting this conclusion were derived from four related but independent animal experiments which were designed to study the effects of continuous shock (CSh) and intermittent shock (ISh) on extinction behavior. The findings being presented in this paper were accidental and secondary observations which became apparent as a result of the sequence of experiments. Experiments 1 (Kolstoe, Kleban, \& Utecht, 1964) and 2 (Kleban, 1965), and Experiments 3 (Kleban, 1967a) and 4 (Kleban, 1967b) are discussed separately in the methods and results sections. \section{METHOD}

Experiments 1 and 2. The experimental designs, apparatus, and procedures used by Kolstoe, Kleban, \& Utecht (1964) and Kleban (1965) are similar. These studies may be referred to for methodological details. To briefly describe the experiments, the apparatus were Y-mazes with 20-in. electric grids in the stem. Forty trials of food-reward training to one arm of the maze were given to 141 albino rats. For Trials $41-100,410 \mathrm{~V}$ electric shocks were given in the stem. CSh Ss received 60 shock trials; ISh Ss received shock trials randomly interspersed with no-shock trials. On Trial 101, shock was discontinued, and the food reward was switched to the unreinforced arm. The Ss' task was to reverse their responses from the preferred arm to the arm containing the food reward. This paper, however, is concerned only with a subgroup of 19 animals that reversed from their food oriented running responses to the nonfood arm when electric shock was introduced on Trials 41-100. Measurements were made of the number of no-shock extinction trials required before these Ss returned to their respective food arms.

Experiments 3 and 4. The apparatus and methodology for these experiments were also similar. The apparatus were $Y$-mazes. In Experiment 3 (Kleban, 1967a), the electric grid was 20 in. long; it was 6 in. long in Experiment 4 (Kleban, 1967b). Food reward was not used in these experiments. Solely on the basis of escape from electric shock 210 albino rats were forced to make stereotyped position running responses. Both experiments had groups of CSh and ISh Ss. Twenty aversive training trials were followed by 40 no-shock extinction trials. The experiments were concerned with extinction performance. The emphasis of this paper, however, is centered on maze arm vacillations occurring before the Ss were coerced by the electric shock to adopt stereotyped running responses. The measures used were: (1) the number of trials before stereotyped responses were adopted; and (2) the number of vacillations made before stereotype responses were adopted.

\section{RESULTS}

Experiments 1 and 2. A percentage of ISh and CSh Ss reversed from their established food responses and consistently ran to the nonrewarded arm when electric shock was introduced. Eleven out of $37 \mathrm{CSh}$ and eight out of 104 ISh Ss made nonfood oriented reversals when shock was introduced. The chi-square was significant $\left(X^{2}=55.03\right.$, df $\left.=1, p<.001\right)$, indicating that CSh produced a greater degree of interference with the food oriented response. During extinction training, the eight ISh Ss returned to their food arm in a mean of 36.12 trials. The $11 \mathrm{CSh}$ Ss returned to their food arms in a mean of 8.82 trials. A U of $6.5, \mathrm{~N}_{1}=8, \mathrm{~N}_{2}=11$, was significant $(\mathrm{p}<.001)$. It appears that $\mathrm{CSh}$ produced more disruptions of food oriented behavior, but food reorientation occurred quickly once shock was terminated. Obversely, ISh produced a lower proportion of food disruptions but the disruptive effect persisted for many more extinction trials.

Experiments 3 and 4 . A 2 by 2 analysis of variance was run on the first criterion, the number of trials before stereotyped responses were adopted. The F-ratio between the ISh $(M=8.25)$ and $\operatorname{CSh}(\mathrm{M}=5.89)$ conditions was significant $(\mathrm{F}=4.24$, df $=$ $1 / 206, p<.05)$. CSh produced a faster rate of response stereotypy. The length of the grid did not contribute to the difference between the ISh and CSh groups.

For the second criterion (number of vacillations made before stereotyped responses were adopted), once again the difference between the ISh $(M=4.28)$ and the CSh $(M=3.25)$ schedules was significant $(F=5.39, \mathrm{df}=1 / 206, \mathrm{p}<.025)$. A significant interaction was also present $(F=3.87, \mathrm{df}=1 / 206, \mathrm{p}<.05)$. The $\mathrm{CSh}, 20$-in. grid condition $(\mathrm{M}=2.68)$ produced faster response stereotypy than: (1) the CSh, 6 -in. grid group $(M=4.00, t=2.49$, $\mathrm{p}<.02) ;(2)$ ISh, 20-in. grid group $(\mathrm{M}=4.48, \mathrm{t}=2.86, \mathrm{p}<.01)$; and (3) ISh, 6-in. grid group $(M=4.02, t=2.73, p<.01)$. The CSh condition exerted the strongest stereotyping effect which was especially pronounced when CSh was combined with the longer grid.

\section{DISCUSSION}

The direction of the results is very clear: (1) CSh is far more distuptive to food oriented behavior than ISh; and (2) CSh also coerces stereotyped running behavior more quickly than ISh. Once the pain experience is removed, however, the ISh groups, under the influence of fear conditioning (refer to: Kleban, 1965, 1967a, b, 1968), show much greater resistance to extinction (GRE).

These observations help throw some light on other experimental settings. The "vicious circle phenomenon" (Brown, Martin, \& Morrow, 1964), makes the animals appear either very stupid or "masochistic." After receiving escape training, with shock in both the start box and stem, the Ss receive shock only in the stem during extinction training. They can avoid shock by standing still in the start box. Yet they continue to tuisi out of the safe start box into the painful portion of the grid. In this case the action of fear conditioning and pain stereotypy work in the same direction. The fear of shock coming on impeis the animals to run forward. This phenomenon occurs with just one shock experience (Kleban, 1965); the vicious-circle Ss have many shock experiences. The running forward leads them into shock, that is, further impelling the running forward behavior in response to shock). Thus, the experimental conditions are such that they cannot possibly learn to stand still to avoid shock. Parenthetically, the Ss could probably learn to avoid shock if they recoiled backward rather than darted forward in response to the electric shock. This would leave us with sume very frightened but extinguished Ss.

These CSh and ISh observations also help to clarify D'Amato, Keller, \& DiCara's (1964) discontinuous shock phenomenon. The disccntinuous shock is produced by fast on-off pulsations of electric shock. The discontinuous-shock Ss evidence GRE more than CSh Ss; that is, they act like fear motivated animals. In his work on ISh, Kleban $(1965,1967 \mathrm{a}, \mathrm{b}, 1968)$ explained the GRE on the basis of fear conditioning (refer to these papers for details of the explanation). Briefly, the critical event is the separation of the fear component from the pain-fear reaction. The ISh schedule allows the Ss to experience their fearfulness independent of pain and in association with their adaptive instrumental activity. Thus the stimuli evoked by the instrumental response can by themselves, through conditioning, elicit the fear reaction, thereby producing GRE of the instrumental activity. The same explanation applies to the discontinuous shock situation. The off pulsation of electric shock allows the Ss to experience fear independent of pain and the adaptive instrumental activity becomes capable of eliciting the fear state through conditioning

\section{REFERENCES}

BROWN, J. S. MARTIN, R. C., \& MORROW, M W. Self punitive behavior in the rat: facilitative effects of punishment on resistance to extinction. $J$. comp. physiol. Psychol., 1964, 57, 127-133. 
D'AMATO, M. R., KELLER, D., \& DICARA, L. Facilitation of discriminated avoidance learning by discontinuous shock. J. comp. physiol. Psychol., 1964, 58, 344-349.

KLEBAN, M. H. The effects of three different types of punishment on the performance of albino rats. J. genet. Psychol., 1965, 106, 15-21.

KLEBAN, M. H. Studies of punishment relating omissions in noxious stimulation to response-reversals. J. genet. Psychol., 1967a, 110, 127-138.

KLEBAN, M. H. A test of the discrimination hypothesis: Utilization of response-reversals to test resistance to extinction for escape and intermit- tent escape conditions. J. genet. Psychol., 1967b, 111, 125-134.

KLEBAN, M. H. A comparison of different types of intermittent shock schedules. Psychon. Sci., 1968, 10, 179-180.

KOLSTOE, R. H., KLEBAN, M. H. \& UTECHT, A. J. The effect of delay in the maintenance of fixated behavior in albino rats. J. genet. Psychol, $1964,105,275-282$.

MILLER, N. E. Studies of fear as an acquirable drive. I. Fear as motivation and fear-reduction as reinforcement in the learning of new responses. $J$. exp. Psychol., 1948, 38, 89-101. 